\title{
ON THE HARMONIC VIBRATIONS IN LINEAR THERMOELASTICITY WITHOUT ENERGY DISSIPATION
}

\author{
S. Chiriţă $\breve{1}^{1}$ and M. Ciarletta ${ }^{2}$ \\ ${ }^{1}$ Faculty of Mathematics, Al. I. Cuza University of Iaşi, Iaşi, Romania \\ ${ }^{2}$ DIIMA, University of Salerno, Fisciano (SA), Italy
}

In the present paper we consider a prismatic cylinder occupied by an anisotropic homogeneous compressible linear thermoelastic material that is subject to zero body force and heat supply and zero displacement and thermal displacement on the lateral boundary. The motion is induced by a harmonic time-dependent displacement and thermal displacement specified pointwise over the base. We establish some spatial estimates for an appropriate cross-sectional measure associated with the amplitude of the corresponding harmonic vibration in the high frequency range that describe how the amplitude evolves with respect to the axial distance to the excited base. In fact, in the high frequency range we establish certain algebraical estimates that predict decay or growth slower than that for the low frequency range.

Keywords: Harmonic vibrations; Spatial behavior; Thermoelasticity without energy dissipation

\section{INTRODUCTION}

In the literature concerning thermal effects in continuum mechanics there are developed several parabolic and hyperbolic theories for describing the heat conduction. The hyperbolic theories are also called theories of second sound and there the flow of heat is modelled with finite propagation speed, in contrast to the classical model based on the Fourier's law leading to infinite propagation speed of heat signals. A review of these theories is presented in the articles by Chandrasekharaiah [1] and Hetnarski and Ignaczak [2, 3].

A new thermoelastic theory without energy dissipation has been proposed by Green and Naghdi [4]. This thermomechanical theory of deformable media introduces the so-called thermal displacement related to the common temperature and uses a general entropy balance as postulated in [5]. By the procedure of Green and Naghdi [6], the reduced energy equation is regarded as an identity for all thermodynamical processes and places some restrictions on the functional forms of the dependent constitutive variables. The theory is illustrated in detail in the context of flow of heat in a rigid solid, with particular reference to the propagation of

Received 4 December 2009; accepted 30 December 2009.

The author SC acknowledges support from the Romanian Ministry of Education and Research and Innovation, CNCSIS under Grant code ID-89, Contract no. 457.

Address correspondence to M. Ciarletta, DIIMA, University of Salerno, Via Ponte Don Melillo, Fisciano, SA 84084, Italy. E-mail: ciarletta@diima.unisa.it 
thermal waves at finite speed. The linearized formulation allows the transmission of heat flow as thermal waves at finite speed and the evolution equations are fully hyperbolic.

The linear theory of thermoelasticity without energy dissipation for homogeneous and isotropic materials was employed by Nappa [7] and Quintanilla [8] to obtain spatial energy bounds and decay estimates for the transient solutions in connection with the problem in which a thermoelastic body is deformed subject to boundary and initial data and body supplies having a compact support, provided positive definiteness assumptions are supposed upon the constitutive coefficients. Moreover, we have to mention that Chandrasekharaiah [9] proves uniqueness of solutions, Ieşan [10] establishes continuous dependence results, while Quintanilla [11] studies the question of existence. Further results of structural stability and decay type are given by Quintanilla [12, 13]. Quintanilla and Straughan [14] used logarithmic convexity and Lagrange identity arguments to yield uniqueness and growth without requiring sign definiteness of the constitutive coefficients, while Quintanilla and Straughan [15] derive energy bounds for a class of non-standard problems in which the initial data are given as a combination of data at initial time and at a later time.

The present paper is concerned with the study of the spatial behavior of the harmonic in time vibrations within the model of the linear thermoelasticity theory without dissipation energy for anisotropic materials. We derive some differential inequalities for certain cross-sectional integrals and integration leads to estimates describing how these integrals evolve with respect to the distance to the excited base, provided the frequency of vibration is greater than a certain critical value. The method employed establishes differential inequalities for the selected measures which after integration provides algebraic estimates for spatial evolution, provided the strong ellipticity of the constitutive coefficients is assumed. However, here we use an idea developed by Chiriţă and Ciarletta [16] for linear anisotropic materials with a strong elliptic elasticity tensor. In fact, we derive a priori estimates for the amplitude of a harmonic vibration by means of some auxiliary identities, some of these being achieved via some Rellich-like identities (used, for example, in [17, 18]). Generally, these estimates predict some algebraic spatial decay or growth properties for the amplitude of the harmonic vibrations. However, there can exist classes of harmonic vibrations for which the spatial decay or growth properties are of exponential type.

In the classical linear thermoelasticity the spatial behavior of the harmonic in time vibrations has been studied by Chiriţă [19] by using a technique developed by Flavin and Knops [20] and Flavin et al. [21] in the low frequency range. There are established some differential inequalities for the appropriate selected measures which after integration provides exponential estimates for spatial evolution of the amplitude of vibration, provided the positive definiteness of the constitutive coefficients is assumed.

\section{NOTATION AND BASIC THEORY}

Throughout this section we assume that the region $\Omega$ is occupied by a homogeneous and anisotropic thermoelastic material with a center of symmetry at 
each point. In what follows we will consider the dynamic theory of thermoelasticity without energy dissipation as described in $[4,6]$.

The governing equations of the linear theory of anisotropic and homogeneous thermoelasticity without energy dissipation are given by the evolution equations $[4,6]$

$$
\begin{gathered}
S_{r s, r}+\rho b_{s}=\rho \ddot{u}_{s} \\
\rho \dot{\eta}=\frac{\rho}{\theta_{0}} r-q_{r, r}
\end{gathered}
$$

in $\Omega \times(0, \infty)$, the constitutive equations

$$
\begin{aligned}
S_{r s} & =C_{r s m n} e_{m n}-M_{r s} \theta \\
\rho \eta & =M_{r s} e_{r s}+\frac{c}{\theta_{0}} \theta \\
q_{r} & =-\frac{1}{\theta_{0}} K_{r s} \beta_{s}
\end{aligned}
$$

in $\bar{\Omega} \times[0, \infty)$, the geometrical equations

$$
e_{r s}=\frac{1}{2}\left(u_{r, s}+u_{s, r}\right)
$$

in $\bar{\Omega} \times[0, \infty)$ and the law of heat flow

$$
\dot{\beta}_{r}=\theta_{, r}
$$

in $\bar{\Omega} \times[0, \infty)$. Here $u_{r}$ are the components of the displacement vector, $\theta$ is the temperature variation from the uniform reference temperature $\theta_{0}, e_{r s}$ are the components of the strain tensor, $\beta_{r}$ are the components of the thermal displacement gradient vector, $S_{r s}$ are the components of the stress tensor, $q_{r}$ are the components of the entropy-heat flux vector, $\eta$ is the entropy density per unit mass and $b_{r}$ represents the components of the external body force vector and $r$ is the external rate of supply of heat per unit mass. Furthermore, $\rho$ is the constant density mass, $C_{r s m n}, M_{r s}, c$ and $K_{r s}$ are the constant constitutive coefficients satisfying the following symmetries

$$
C_{r s m n}=C_{m n r s}=C_{s r m n}, \quad M_{r s}=M_{s r}, \quad K_{r s}=K_{s r}
$$

and the subscripts $r, s, m, n$ take values 1,2, 3 and summation is implied by index repetition. Moreover, a superposed dot denotes differentiation with respect to time and a subscript comma indicates partial differentiation. The specific Helmholtz free energy $\psi$ is given by

$$
\rho \psi=\frac{1}{2} C_{r s m n} e_{r s} e_{m n}-M_{r s} e_{r s} \theta-\frac{c}{2 \theta_{0}} \theta^{2}+\frac{1}{2 \theta_{0}} K_{r s} \tau_{, r} \tau_{, s}
$$


while the specific internal energy $\epsilon$ is given by

$$
\rho \epsilon=\frac{1}{2} C_{r s m n} e_{r s} e_{m n}+\frac{c}{2 \theta_{0}} \theta^{2}+\frac{1}{2 \theta_{0}} K_{r s} \tau_{, r} \tau_{, s}
$$

where $\tau$ is the thermal displacement related to the temperature variation by relation

$$
\dot{\tau}=\theta
$$

In view of the relations (1)-(6) and (9) we can express the basic field equations in terms of the displacements $u_{r}$ and the thermal displacement $\tau$ as

$$
\begin{gathered}
\left(C_{r s m n} u_{m, n}-\dot{M}_{r s} \dot{\tau}\right)_{, r}+\rho b_{s}=\rho \ddot{u}_{s} \\
\frac{1}{\theta_{0}}\left(K_{r s} \tau_{, s}\right)_{, r}-M_{r s} \dot{u}_{r, s}+\frac{\rho}{\theta_{0}} r=\frac{c}{\theta_{0}} \ddot{\tau}
\end{gathered}
$$

in $\Omega \times(0, \infty)$. Clearly, the present theory permits propagation of heat as thermal waves at finite speed.

\section{HARMONIC VIBRATIONS OF A CYLINDER}

Throughout in the remainder of the present paper we assume that $\Omega$ is the prismatic cylinder $B \subset \mathbb{R}^{3}$ whose bounded uniform cross-section $D \subset \mathbb{R}^{2}$ has piecewise continuously differentiable boundary $\partial D$. The origin of the rectangular Cartesian coordinate system is located in the cylinder's base and the positive $x_{3}$-axis is directed along that of the cylinder. Then the lateral boundary is $\pi=\partial D \times[0, L]$, where $L$ is the length of the cylinder. The cylinder is occupied by a homogeneous and anisotropic linear thermoelastic solid with a center of symmetry. It is subjected to zero body force and heat supply, zero displacement and thermal displacement on the lateral boundary surface and pointwise specified harmonic in time displacement and thermal displacement over the base. Thus, to the basic system of differential equations (10) we adjoin the lateral boundary conditions

$$
u_{s}(x, t)=0, \quad \tau(x, t)=0, \quad(x, t) \in \pi \times(0, \infty)
$$

and the base boundary conditions

$u_{s}\left(x_{1}, x_{2}, 0, t\right)=\tilde{v}_{s}\left(x_{1}, x_{2}\right) e^{i \omega t}, \quad \tau\left(x_{1}, x_{2}, 0, t\right)=\widetilde{T}\left(x_{1}, x_{2}\right) e^{i \omega t}, \quad\left(x_{1}, x_{2}\right) \in D(0), \quad t>0$

where $i=\sqrt{-1}$ is the complex unit, $\tilde{v}_{s}\left(x_{1}, x_{2}\right)$ and $\widetilde{T}\left(x_{1}, x_{2}\right)$ are prescribed smooth functions and $\omega$ is a prescribed positive constant. Other types of boundary conditions can be also considered.

The above given data lead to a harmonic in time vibration inside the cylinder, having the form

$$
u_{s}\left(x_{1}, x_{2}, x_{3}, t\right)=v_{s}\left(x_{1}, x_{2}, x_{3}\right) e^{i \omega t}, \quad \tau\left(x_{1}, x_{2}, x_{3}, t\right)=T\left(x_{1}, x_{2}, x_{3}\right) e^{i \omega t}
$$


whose amplitude $\left(v_{s}, T\right)(x)$ satisfies the following differential system

$$
\begin{gathered}
\left(C_{r s m n} v_{m, n}-i \omega M_{r s} T\right)_{, r}+\rho \omega^{2} v_{s}=0 \\
\left(\frac{1}{\theta_{0}} K_{r s} T_{, s}\right)_{, r}-i \omega M_{r s} v_{r, s}+\frac{c}{\theta_{0}} \omega^{2} T=0
\end{gathered}
$$

subjected to the lateral boundary conditions

$$
v_{s}(x)=0, \quad T(x)=0, \quad x \in \pi
$$

and the base boundary conditions

$$
v_{s}\left(x_{1}, x_{2}, 0\right)=\tilde{v}_{s}\left(x_{1}, x_{2}\right), \quad T\left(x_{1}, x_{2}, 0\right)=\widetilde{T}\left(x_{1}, x_{2}\right), \quad\left(x_{1}, x_{2}\right) \in D(0)
$$

When a finite cylinder will be considered then a boundary condition has to be considered on $D(L)$. For our next analysis we have no need to write it explicitly. The spatial behavior of the amplitude $\left(v_{r}, T\right)$ of the forced oscillation has been established in [22] for the case of rhombic thermoelastic materials, provided the exciting frequency is less than a certain critical frequency.

In what follows we will establish some spatial estimates describing how the amplitude evolves with respect to the axial distance to the excited end under the assumption of strong ellipticity condition upon the elasticity tensor in the high frequency range.

\section{SOME AUXILIARY IDENTITIES}

Before proceeding to derive a priori estimates for a solution to equations (14)-(17), we need some auxiliary identities concerning the equations (14) and (15), with the lateral boundary condition described in (16). These are achieved via the use of Rellich-like identities (used, for example, in [17, 18]).

Theorem 1. Let $\left(v_{r}, T\right)$ be a solution of the boundary value problem defined by relations (14)-(16). Then we have the following identities

$$
\begin{aligned}
& 2 \int_{D\left(x_{3}\right)}\left(C_{r s m n} v_{r, s} \bar{v}_{m, n}-\rho \omega^{2} v_{s} \bar{v}_{s}\right) d a+\int_{D\left(x_{3}\right)} i \omega M_{r s}\left(\bar{T} v_{r, s}-T \bar{v}_{r, s}\right) d a \\
& \quad=\frac{d}{d x_{3}} \int_{D\left(x_{3}\right)}\left[\left(C_{3 s m n} v_{m, n}-i \omega M_{3 s} T\right) \bar{v}_{s}+\left(C_{3 s m n} \bar{v}_{m, n}+i \omega M_{3 s} \bar{T}\right) v_{s}\right] d a \\
& \int_{D\left(x_{3}\right)} i \omega M_{r s}\left(\bar{v}_{r, s} T+v_{r, s} \bar{T}\right) d a \\
& \quad=\frac{d}{d x_{3}} \int_{D\left(x_{3}\right)}\left[\left(C_{3 s m n} \bar{v}_{m, n}+i \omega M_{3 s} \bar{T}\right) v_{s}-\left(C_{3 s m n} v_{m, n}-i \omega M_{3 s} T\right) \bar{v}_{s}\right] d a \\
& 2 \int_{D\left(x_{3}\right)} \frac{1}{\theta_{0}}\left(K_{r s} T_{, r} \bar{T}_{, s}-c \omega^{2} T \bar{T}\right) d a+\int_{D\left(x_{3}\right)} i \omega M_{r s}\left(\bar{T} v_{r, s}-T \bar{v}_{r, s}\right) d a \\
& \quad=\frac{d}{d x_{3}} \int_{D\left(x_{3}\right)} \frac{1}{\theta_{0}} K_{33}\left(T \bar{T}_{, 3}+T_{, 3} \bar{T}\right) d a
\end{aligned}
$$




$$
\int_{D\left(x_{3}\right)} i \omega M_{r s}\left(T \bar{v}_{r, s}+\bar{T} v_{r, s}\right) d a=\frac{d}{d x_{3}} \int_{D\left(x_{3}\right)} \frac{1}{\theta_{0}} K_{3 s}\left(\bar{T} T_{, s}-T \bar{T}_{, s}\right) d a
$$

where a superposed bar over a quantity denotes the complex conjugate of that quantity.

Proof. We form the identity

$$
\bar{v}_{s}\left[\left(C_{r s k l} v_{k, l}-i \omega M_{r s} T\right)_{, r}+\rho \omega^{2} v_{s}\right]+v_{s}\left[\left(C_{r s k l} \bar{v}_{k, l}+i \omega M_{r s} \bar{T}\right)_{, r}+\rho \omega^{2} \bar{v}_{s}\right]=0
$$

which can be written as

$$
\begin{aligned}
& 2\left(C_{r s m n} v_{r, s} \bar{v}_{m, n}-\rho \omega^{2} v_{s} \bar{v}_{s}\right)+i \omega M_{r s}\left(\bar{T} v_{r, s}-T \bar{v}_{r, s}\right) \\
& \quad=\left[\left(C_{r s m n} v_{m, n}-i \omega M_{r s} T\right) \bar{v}_{s}+\left(C_{r s m n} \bar{v}_{m, n}+i \omega M_{r s} \bar{T}\right) v_{s}\right]_{, r}
\end{aligned}
$$

Then we integrate over $D\left(x_{3}\right)$ and use the lateral boundary condition described in (16) to find relation (18).

Furthermore, we continue with the identity

$$
\bar{v}_{s}\left[\left(C_{r s m n} v_{m, n}-i \omega M_{r s} T\right)_{, r}+\rho \omega^{2} v_{s}\right]-v_{s}\left[\left(C_{r s m n} \bar{v}_{m, n}+i \omega M_{r s} \bar{T}\right)_{, r}+\rho \omega^{2} \bar{v}_{s}\right]=0
$$

which leads to

$$
i \omega M_{r s}\left(\bar{v}_{r, s} T+v_{r, s} \bar{T}\right)=\left[\left(C_{r s m n} \bar{v}_{m, n}+i \omega M_{r s} \bar{T}\right) v_{s}-\left(C_{r s m n} v_{m, n}-i \omega M_{r s} T\right) \bar{v}_{s}\right]_{, r}
$$

After an integration over $D\left(x_{3}\right)$ and the use of the lateral boundary condition described in (16), we deduce relation (19).

Now we consider

$$
\bar{T}\left[\left(\frac{1}{\theta_{0}} K_{r s} T_{, s}\right)_{, r}-i \omega M_{r s} v_{r, s}+\frac{c}{\theta_{0}} \omega^{2} T\right]+T\left[\left(\frac{1}{\theta_{0}} K_{r s} \bar{T}_{, s}\right)_{, r}+i \omega M_{r s} \bar{v}_{r, s}+\frac{c}{\theta_{0}} \omega^{2} \bar{T}\right]=0
$$

which gives

$$
\frac{2}{\theta_{0}}\left(K_{r s} T_{, r} \bar{T}_{, s}-c \omega^{2} T \bar{T}\right)+i \omega M_{r s}\left(\bar{T} v_{r, s}-T \bar{v}_{r, s}\right)=\left[\frac{1}{\theta_{0}} K_{r s}\left(\bar{T} T_{, s}+T \bar{T}_{, s}\right)\right]_{, r}
$$

and hence, by an integration over $D\left(x_{3}\right)$ and the use of the lateral boundary condition described in (16), we obtain relation (20).

Finally, we consider

$$
\bar{T}\left[\left(\frac{1}{\theta_{0}} K_{r s} T_{, s}\right)_{, r}-i \omega M_{r s} v_{r, s}+\frac{c}{\theta_{0}} \omega^{2} T\right]-T\left[\left(\frac{1}{\theta_{0}} K_{r s} \bar{T}_{, s}\right)_{, r}+i \omega M_{r s} \bar{v}_{r, s}+\frac{c}{\theta_{0}} \omega^{2} \bar{T}\right]=0
$$

so that we obtain

$$
i \omega M_{r s}\left(T \bar{v}_{r, s}+\bar{T} v_{r, s}\right)=\left[\frac{1}{\theta_{0}} K_{r s}\left(\bar{T} T_{, s}-T \bar{T}_{, s}\right)\right]_{, r}
$$


Then, by an integration of (29) over $D\left(x_{3}\right)$ and the use of the lateral boundary condition expressed in (16), we obtain relation (21) and the proof is complete.

Theorem 2. Let $\left(v_{r}, T\right)$ be a solution of the boundary value problem defined by relations (14)-(16). Then we have the following Rellich type identities

$$
\begin{gathered}
\int_{D\left(x_{3}\right)}\left(C_{r s m n} v_{r, s} \bar{v}_{m, n}-3 \rho \omega^{2} v_{s} \bar{v}_{s}\right) d a-\int_{D\left(x_{3}\right)} i \omega\left[2 M_{r s}\left(T \bar{v}_{r, s}-\bar{T} v_{r, s}\right)\right. \\
\left.+M_{r s} x_{p}\left(T_{, p} \bar{v}_{r, s}-\bar{T}_{, p} v_{r, s}\right)\right] d a+\int_{\partial D\left(x_{3}\right)} x_{\rho} n_{\rho} C_{r \alpha m \beta} n_{\alpha} n_{\beta} \frac{\partial v_{r}}{\partial n} \frac{\partial \bar{v}_{m}}{\partial n} d s \\
=-\frac{d}{d x_{3}} \int_{D\left(x_{3}\right)}\left\{x_{\rho} \bar{v}_{s, \rho}\left(C_{3 s m n} v_{m, n}-i \omega M_{3 s} T\right)+x_{\rho} v_{s, \rho}\left(C_{3 s m n} \bar{v}_{m, n}+i \omega M_{3 s} \bar{T}\right)\right. \\
\left.+x_{3}\left[C_{r 3 m 3} v_{r, 3} \bar{v}_{m, 3}-C_{r \alpha m \beta} v_{r, \alpha} \bar{v}_{m, \beta}+i \omega M_{r \alpha}\left(T \bar{v}_{r, \alpha}-\bar{T} v_{r, \alpha}\right)+\rho \omega^{2} v_{s} \bar{v}_{s}\right]\right\} d a \\
\int_{D\left(x_{3}\right)} \frac{1}{\theta_{0}}\left(K_{r s} T_{, r} \bar{T}_{, s}-3 c \omega^{2} T \bar{T}\right) d a+\int_{D\left(x_{3}\right)} i \omega M_{r s} x_{p}\left(T_{, p} \bar{v}_{r, s}-\bar{T}_{, p} v_{r, s}\right) d a \\
+\int_{\partial D\left(x_{3}\right)} \frac{1}{\theta_{0}} x_{\rho} n_{\rho} K_{\alpha \beta} n_{\alpha} n_{\beta} \frac{\partial T}{\partial n} \frac{\partial \bar{T}}{\partial n} d s \\
=-\frac{d}{d x_{3}} \int_{D\left(x_{3}\right)}\left\{\frac{1}{\theta_{0}}\left[x_{\alpha} K_{3 \beta}\left(\bar{T}_{, \alpha} T_{, \beta}+T_{, \alpha} \bar{T}_{, \beta}\right)+x_{\alpha} K_{33}\left(T_{, 3} \bar{T}_{, \alpha}+\bar{T}_{, 3} T_{, \alpha}\right)\right]\right. \\
\left.+\frac{x_{3}}{\theta_{0}}\left(K_{33} T_{, 3} \bar{T}_{, 3}-K_{\alpha \beta} T_{, \alpha} \bar{T}_{, \beta}+c \omega^{2} T \bar{T}\right)\right\} d a
\end{gathered}
$$

where $n_{\alpha}$ are the components of the outward unit normal vector to $\partial D$ and $(\partial / \partial n)$ represents the normal derivative.

Proof. We start with the identity

$$
x_{p} \bar{v}_{s, p}\left[\left(C_{r s m n} v_{m, n}-i \omega M_{r s} T\right)_{, r}+\rho \omega^{2} v_{s}\right]+x_{p} v_{s, p}\left[\left(C_{r s m n} \bar{v}_{m, n}+i \omega M_{r s} \bar{T}\right)_{, r}+\rho \omega^{2} \bar{v}_{s}\right]=0
$$

which furnishes

$$
\begin{aligned}
& {\left[x_{p} \bar{v}_{s, p}\left(C_{r s m n} v_{m, n}-i \omega M_{r s} T\right)+x_{p} v_{s, p}\left(C_{r s m n} \bar{v}_{m, n}+i \omega M_{r s} \bar{T}\right)\right]_{, r}-2 C_{r s m n} v_{r, s} \bar{v}_{m, n}} \\
& \quad+i \omega M_{r s}\left(T \bar{v}_{r, s}-\bar{T} v_{r, s}\right)-x_{p}\left(C_{r s m n} v_{r, s} \bar{v}_{m, n}\right)_{, p}+i \omega x_{p} M_{r s}\left(T \bar{v}_{r, s p}-\bar{T} v_{r, s p}\right) \\
& \quad+x_{p}\left(\rho \omega^{2} v_{s} \bar{v}_{s}\right)_{, p}=0
\end{aligned}
$$

and moreover, we obtain

$$
\begin{aligned}
& C_{r s m n} v_{r, s} \bar{v}_{m, n}-3 \rho \omega^{2} v_{s} \bar{v}_{s}-2 i \omega M_{r s}\left(T \bar{v}_{r, s}-\bar{T} v_{r, s}\right)-i \omega x_{p} M_{r s}\left(T_{, p} \bar{v}_{r, s}-\bar{T}_{, p} v_{r, s}\right) \\
& =-\left[x_{p} \bar{v}_{s, p}\left(C_{r s m n} v_{m, n}-i \omega M_{r s} T\right)+x_{p} v_{s, p}\left(C_{r s m n} \bar{v}_{m, n}+i \omega M_{r s} \bar{T}\right)\right]_{, r} \\
& \quad+\left[x_{p} C_{r s m n} v_{r, s} \bar{v}_{m, n}-i \omega x_{p} M_{r s}\left(T \bar{v}_{r, s}-\bar{T} v_{r, s}\right)-x_{p} \rho \omega^{2} v_{s} \bar{v}_{s}\right]_{, p}
\end{aligned}
$$


Furthermore, by direct integration over $D\left(x_{3}\right)$ and the use of the lateral boundary condition in (16), we obtain

$$
\begin{aligned}
& \int_{D\left(x_{3}\right)}\left(C_{r s m n} v_{r, s} \bar{v}_{m, n}-3 \rho \omega^{2} v_{s} \bar{v}_{s}\right) d a \\
& -\int_{D\left(x_{3}\right)}\left[2 i \omega M_{r s}\left(T \bar{v}_{r, s}-\bar{T} v_{r, s}\right)+i \omega M_{r s} x_{p}\left(T_{, p} \bar{v}_{r, s}-\bar{T}_{, p} v_{r, s}\right)\right] d a \\
& =-\frac{d}{d x_{3}} \int_{D\left(x_{3}\right)}\left\{x_{p} \bar{v}_{s, p}\left(C_{3 s m n} v_{m, n}-i \omega M_{3 s} T\right)+x_{p} v_{s, p}\left(C_{3 s m n} \bar{v}_{m, n}+i \omega M_{3 s} \bar{T}\right)\right. \\
& \left.\quad+x_{3}\left[\rho \omega^{2} v_{s} \bar{v}_{s}+i \omega M_{r s}\left(T \bar{v}_{r, s}-\bar{T} v_{r, s}\right)-C_{r s m n} v_{r, s} \bar{v}_{m, n}\right]\right\} d a \\
& -\int_{\partial D\left(x_{3}\right)}\left(x_{p} \bar{v}_{s, p} C_{\rho s m n} v_{m, n}+x_{p} v_{s, p} C_{\rho s m n} \bar{v}_{m, n}\right) n_{\rho} d s+\int_{\partial D\left(x_{3}\right)} x_{\rho} n_{\rho} C_{r s m n} v_{r, s} \bar{v}_{m, n} d s
\end{aligned}
$$

We now use the lateral boundary condition described in (16) to infer that

$$
v_{r, 3}=0 \text { on } \partial D\left(x_{3}\right)
$$

Further, we write $v_{r, \alpha}$ on the curve $\partial D$ as $v_{r, \alpha}=n_{\alpha} \frac{\partial v_{r}}{\partial n}+\tau_{\alpha} \frac{\partial v_{r}}{\partial \tau}$, where $\tau_{\alpha}$ are the components of the unit vector tangent to $\partial D$ and $\frac{\partial}{\partial \tau}$ is the tangential derivative. If we recall the lateral boundary condition in (16), then we can deduce that $\frac{\partial v_{r}}{\partial \tau}=0$ on the curve $\partial D$ and hence we have

$$
v_{r, \alpha}=n_{\alpha} \frac{\partial v_{r}}{\partial n} \text { on the curve } \partial D
$$

By using relations (36) and (37), we obtain

$$
\int_{\partial D\left(x_{3}\right)} x_{\rho} n_{\rho} C_{r s m n} v_{r, s} \bar{v}_{m, n} d s=\int_{\partial D\left(x_{3}\right)} x_{\rho} n_{\rho} C_{r \alpha m \beta} n_{\alpha} n_{\beta} \frac{\partial v_{r}}{\partial n} \frac{\partial \bar{v}_{m}}{\partial n} d s
$$

and

$$
-\int_{\partial D\left(x_{3}\right)}\left(x_{p} \bar{v}_{s, p} C_{\rho s m n} v_{m, n}+x_{p} v_{s, p} C_{\rho s m n} \bar{v}_{m, n}\right) n_{\rho} d s=-2 \int_{\partial D\left(x_{3}\right)} x_{\rho} n_{\rho} C_{r \alpha m \beta} n_{\alpha} n_{\beta} \frac{\partial v_{r}}{\partial n} \frac{\partial \bar{v}_{m}}{\partial n} d s
$$

Moreover, we have

$$
\begin{aligned}
\int_{D\left(x_{3}\right)}\left\{x_{3} \bar{v}_{s, 3}\left(C_{3 s m n} v_{m, n}-i \omega M_{3 s} T\right)+x_{3} v_{s, 3}\left(C_{3 s m n} \bar{v}_{m, n}+i \omega M_{3 s} \bar{T}\right)\right. \\
\left.\quad+x_{3}\left[\rho \omega^{2} v_{s} \bar{v}_{s}-i \omega M_{r s}\left(T \bar{v}_{r, s}-\bar{T} v_{r, s}\right)-C_{r s m n} v_{r, s} \bar{v}_{m, n}\right]\right\} d a \\
=\int_{D\left(x_{3}\right)} x_{3}\left[C_{r 3 m 3} v_{r, 3} \bar{v}_{m, 3}-C_{r \alpha m \beta} v_{r, \alpha} \bar{v}_{m, \beta}+i \omega M_{r \alpha}\left(T \bar{v}_{r, \alpha}-\bar{T} v_{r, \alpha}\right)+\rho \omega^{2} v_{s} \bar{v}_{s}\right] d a(40)
\end{aligned}
$$

Concluding, by the substitution of relations (38), (39) and (40) into (35), we obtain relation (30). 
Further, we use the equation (15) to obtain

$$
\begin{aligned}
i \omega M_{r s} x_{p}\left(T_{, p} \bar{v}_{r, s}-\bar{T}_{, p} v_{r, s}\right)= & -x_{p}\left(\frac{c}{\theta_{0}} \omega^{2} T \bar{T}\right)_{, p}-\left[\frac{1}{\theta_{0}} x_{p} K_{r s}\left(\bar{T}_{, p} T_{, s}+T_{, p} \bar{T}_{, s}\right)\right]_{, r} \\
& +\frac{2}{\theta_{0}} K_{r s} T_{, r} \bar{T}_{, s}+x_{p}\left(\frac{1}{\theta_{0}} K_{r s} T_{, r} \bar{T}_{, s}\right)_{, p}
\end{aligned}
$$

and moreover, we have

$$
\begin{aligned}
& \frac{1}{\theta_{0}} K_{r s} T_{, r} \bar{T}_{, s}-\frac{3 c}{\theta_{0}} \omega^{2} T \bar{T}+i \omega M_{r s} x_{p}\left(T_{, p} \bar{v}_{r, s}-\bar{T}_{, p} v_{r, s}\right) \\
& \quad=-\left(\frac{c}{\theta_{0}} x_{p} \omega^{2} T \bar{T}\right)_{, p}-\left[\frac{1}{\theta_{0}} x_{p} K_{r s}\left(\bar{T}_{, p} T_{, s}+T_{, p} \bar{T}_{, s}\right)\right]_{, r}+\left(\frac{x_{p}}{\theta_{0}} K_{r s} T_{, r} \bar{T}_{, s}\right)_{, p}
\end{aligned}
$$

A direct integration on $D\left(x_{3}\right)$ and the use of the lateral boundary condition given in (16) leads to

$$
\begin{aligned}
\int_{D\left(x_{3}\right)} & \frac{1}{\theta_{0}}\left(K_{r s} T_{, r} \bar{T}_{, s}-3 c \omega^{2} T \bar{T}\right) d a+\int_{D\left(x_{3}\right)} i \omega x_{p} M_{r s}\left(T_{, p} \bar{v}_{r, s}-\bar{T}_{, p} v_{r, s}\right) d a \\
= & -\frac{d}{d x_{3}} \int_{D\left(x_{3}\right)}\left[\frac{1}{\theta_{0}} x_{p} K_{3 s}\left(\bar{T}_{, p} T_{, s}+T_{, p} \bar{T}_{, s}\right)-\frac{x_{3}}{\theta_{0}} K_{r s} T_{, r} \bar{T}_{, s}+\frac{x_{3}}{\theta_{0}} c \omega^{2} T \bar{T}\right] d a \\
& +\int_{\partial D\left(x_{3}\right)} \frac{1}{\theta_{0}}\left[x_{\rho} n_{\rho} K_{r s} T_{, r} \bar{T}_{, s}-x_{p} K_{\rho s}\left(\bar{T}_{, p} T_{, s}+T_{, p} \bar{T}_{, s}\right) n_{\rho}\right] d s
\end{aligned}
$$

Since the lateral boundary condition in (16) implies $T_{, 3}=0$ and $T_{, \alpha}=n_{\alpha} \frac{\partial T}{\partial n}$ on $\partial D\left(x_{3}\right)$ it follows that the relation (43) leads to the identity expressed by relation (31) and thus, the proof of theorem is complete.

Theorem 3. Let $\left(v_{r}, T\right)$ be a solution of the boundary value problem defined by relations (14)-(16). Then we have the following conservation laws

$$
\begin{aligned}
\frac{d}{d x_{3}} \int_{D\left(x_{3}\right)} & {\left[\omega^{2}\left(\rho v_{s} \bar{v}_{s}+\frac{c}{\theta_{0}} T \bar{T}\right)+C_{r 3 m 3} v_{r, 3} \bar{v}_{m, 3}+\frac{1}{\theta_{0}} K_{33} T_{, 3} \bar{T}_{, 3}\right.} \\
& \left.-\left(C_{r \alpha m \beta} v_{r, \alpha} \bar{v}_{m, \beta}+\frac{1}{\theta_{0}} K_{\alpha \beta} T_{, \alpha} \bar{T}_{, \beta}\right)+i \omega M_{r \alpha}\left(T \bar{v}_{r, \alpha}-\bar{T} v_{r, \alpha}\right)\right] d a=0 \\
\frac{d}{d x_{3}} \int_{D\left(x_{3}\right)}[ & \left(C_{3 s m n} v_{m, n}-i \omega M_{3 s} T\right) \bar{v}_{s}-\left(C_{3 s m n} \bar{v}_{m, n}+i \omega M_{3 s} \bar{T}\right) v_{s} \\
+ & \left.\frac{1}{\theta_{0}} K_{3 s}\left(\bar{T} T_{, s}-T \bar{T}_{, s}\right)\right] d a=0
\end{aligned}
$$

Proof. We start with the identity

$$
\bar{v}_{s, 3}\left(C_{r s m n} v_{m, n r}-i \omega M_{r s} T_{, r}+\rho \omega^{2} v_{s}\right)+v_{s, 3}\left(C_{r s m n} \bar{v}_{m, n r}-i \omega M_{r s} \bar{T}_{, r}+\rho \omega^{2} \bar{v}_{s}\right)=0
$$


which gives

$$
\begin{aligned}
\frac{d}{d x_{3}} & {\left[\rho \omega^{2} v_{s} \bar{v}_{s}+C_{r 3 m 3} v_{r, 3} \bar{v}_{m, 3}-C_{r \alpha m \beta} v_{r, \alpha} \bar{v}_{m, \beta}+i \omega M_{r \alpha}\left(T \bar{v}_{r, \alpha}-\bar{T} v_{r, \alpha}\right)\right] } \\
& +\left(C_{r \alpha m 3} v_{m, 3} \bar{v}_{r, 3}\right)_{, \alpha}+\left(C_{r \alpha m 3} \bar{v}_{m, 3} v_{r, 3}\right)_{, \alpha}+\left[i \omega M_{r, \alpha}\left(\bar{T} v_{r, 3}-T \bar{v}_{r, 3}\right)\right]_{, \alpha} \\
& +i \omega M_{r s}\left(\bar{T}_{, 3} v_{r, s}-T_{, 3} \bar{v}_{r, s}\right)=0
\end{aligned}
$$

After an integration over $D\left(x_{3}\right)$ and the use of the lateral boundary condition (16), we deduce

$$
\begin{aligned}
& \frac{d}{d x_{3}} \int_{D\left(x_{3}\right)}\left[\rho \omega^{2} v_{s} \bar{v}_{s}+C_{r 3 m 3} v_{r, 3} \bar{v}_{m, 3}-C_{r \alpha m \beta} v_{r, \alpha} \bar{v}_{m, \beta}+i \omega M_{r \alpha}\left(T \bar{v}_{r, \alpha}-\bar{T} v_{r, \alpha}\right)\right] d a \\
& \quad+\int_{D\left(x_{3}\right)} i \omega M_{r s}\left(\bar{T}_{, 3} v_{r, s}-T_{, 3} \bar{v}_{r, s}\right) d a=0
\end{aligned}
$$

Further, we consider

$$
\bar{T}_{, 3}\left(\frac{1}{\theta_{0}} K_{r s} T_{, r s}-i \omega M_{r s} v_{r, s}+\frac{c}{\theta_{0}} \omega^{2} T\right)+T_{, 3}\left(\frac{1}{\theta_{0}} K_{r s} \bar{T}_{, r s}+i \omega M_{r s} \bar{v}_{r, s}+\frac{c}{\theta_{0}} \omega^{2} \bar{T}\right)=0
$$

and hence we get

$$
\begin{aligned}
& \frac{d}{d x_{3}}\left(\frac{c}{\theta_{0}} \omega^{2} T \bar{T}+\frac{1}{\theta_{0}} K_{33} T_{, 3} \bar{T}_{, 3}-\frac{1}{\theta_{0}} K_{\alpha \beta} T_{, \alpha} \bar{T}_{, \beta}\right)+\left(\frac{2}{\theta_{0}} K_{\alpha 3} T_{, 3} \bar{T}_{, 3}\right)_{, \alpha} \\
& \quad+i \omega M_{r s}\left(T_{, 3} \bar{v}_{r, s}-\bar{T}_{, 3} v_{r, s}\right)=0
\end{aligned}
$$

Thus, by an integration over $D\left(x_{3}\right)$ and the use of the lateral boundary condition (16), we obtain

$$
\begin{aligned}
& \frac{d}{d x_{3}} \int_{D\left(x_{3}\right)}\left(\frac{c}{\theta_{0}} \omega^{2} T \bar{T}+\frac{1}{\theta_{0}} K_{33} T_{, 3} \bar{T}_{, 3}-\frac{1}{\theta_{0}} K_{\alpha \beta} T_{, \alpha} \bar{T}_{, \beta}\right) d a \\
& \quad+\int_{D\left(x_{3}\right)} i \omega M_{r s}\left(T_{, 3} \bar{v}_{r, s}-\bar{T}_{, 3} v_{r, s}\right) d a=0
\end{aligned}
$$

Concluding, from relations (48) and (51) we obtain relation (44). The relation (45) is a direct consequence of the relations (19) and (21). Thus, the proof is complete.

The preceding identities can be coupled in various ways leading to different measures associated with the amplitude $\left(v_{r}, T\right)$. Such measures allow us to get appropriate spatial estimates describing the spatial behavior of the corresponding amplitude. We now outline some of these combinations.

A combination of the relations (18) and (20) gives the following result.

Corollary 1. Let $\left(v_{r}, T\right)$ be a solution of the boundary value problem defined by relations (14)-(16). Then we have the following identity

$$
\begin{aligned}
& 2 \int_{D\left(x_{3}\right)}\left[C_{r s m n} v_{r, s} \bar{v}_{m, n}+\frac{1}{\theta_{0}} K_{r s} T_{, r} \bar{T}_{, s}-\omega^{2}\left(\rho v_{s} \bar{v}_{s}+\frac{c}{\theta_{0}} T \bar{T}\right)\right] d a \\
& \quad+2 \int_{D\left(x_{3}\right)} i \omega M_{r s}\left(\bar{T} v_{r, s}-T \bar{v}_{r, s}\right) d a
\end{aligned}
$$




$$
\begin{aligned}
& =\frac{d}{d x_{3}} \int_{D\left(x_{3}\right)}\left[\left(C_{3 s m n} v_{m, n}-i \omega M_{3 s} T\right) \bar{v}_{s}+\left(C_{3 s m n} \bar{v}_{m, n}+i \omega M_{3 s} \bar{T}\right) v_{s}\right. \\
& \left.+\frac{1}{\theta_{0}} K_{33}\left(T \bar{T}_{, 3}+\bar{T} T_{, 3}\right)\right] d a
\end{aligned}
$$

Such identity was used in [22] to establish some exponential estimates describing the spatial evolution of the amplitude of vibration in an appropriate low frequency range. result.

By combining the identities (30), (31) and (52) we obtain the following useful

Corollary 2. Let $\left(v_{r}, T\right)$ be a solution of the boundary value problem defined by relations (14)-(16). Then we have the following identity

$$
\begin{aligned}
& \int_{D\left(x_{3}\right)}\left[C_{r s m n} v_{r, s} \bar{v}_{m, n}\right.\left.+\frac{1}{\theta_{0}} K_{r s} T_{, r} \bar{T}_{, s}+\omega^{2}\left(\rho v_{s} \bar{v}_{s}+\frac{c}{\theta_{0}} T \bar{T}\right)\right] d a \\
&-\int_{\partial D\left(x_{3}\right)} x_{\rho} n_{\rho} C_{r \alpha m \beta} n_{\alpha} n_{\beta} \frac{\partial v_{r}}{\partial n} \frac{\partial \bar{v}_{m}}{\partial n} d s-\int_{\partial D\left(x_{3}\right)} \frac{1}{\theta_{0}} x_{\rho} n_{\rho} K_{\alpha \beta} n_{\alpha} n_{\beta} \frac{\partial T}{\partial n} \frac{\partial \bar{T}}{\partial n} d s \\
&=\frac{d}{d x_{3}} \int_{D\left(x_{3}\right)}\left\{\left(C_{3 s m n} v_{m, n}-i \omega M_{3 s} T\right)\left(\bar{v}_{s}+x_{\rho} \bar{v}_{s, \rho}\right)\right. \\
&+\left(C_{3 s m n} \bar{v}_{m, n}+i \omega M_{3 s} \bar{T}\right)\left(v_{s}+x_{\rho} v_{s, \rho}\right)+\frac{1}{\theta_{0}} K_{33}\left(\bar{T} T_{, 3}+T \bar{T}_{, 3}\right) \\
&+\frac{x_{\alpha}}{\theta_{0}}\left[K_{3 \beta}\left(\bar{T}_{, \alpha} T_{, \beta}+T_{, \alpha} \bar{T}_{, \beta}\right)+K_{33}\left(\bar{T}_{, \alpha} T_{, 3}+T_{, \alpha} \bar{T}_{, 3}\right)\right] \\
&+x_{3}\left[C_{r 3 m 3} v_{r, 3} \bar{v}_{m, 3}-C_{r \alpha m \beta} v_{r, \alpha} \bar{v}_{m, \beta}+i \omega M_{r \alpha}\left(T \bar{v}_{r, \alpha}-\bar{T} v_{r, \alpha}\right)\right. \\
&\left.\left.+\frac{1}{\theta_{0}}\left(K_{33} T_{, 3} \bar{T}_{, 3}-K_{\alpha \beta} T_{, \alpha} \bar{T}_{, \beta}\right)+\omega^{2}\left(\rho v_{s} \bar{v}_{s}+\frac{c}{\theta_{0}} T \bar{T}\right)\right]\right\} d a
\end{aligned}
$$

Throughout this paper we will study the spatial evolution of the amplitude $\left(v_{r}, T\right)$ by starting with the identity (53). To this end we assume that the elasticity tensor satisfies the strong ellipticity condition

$$
C_{r s m n} \xi_{r} \xi_{m} \zeta_{s} \zeta_{n}>0 \text { for all non-zero vectors }\left(\xi_{1}, \xi_{2}, \xi_{3}\right),\left(\zeta_{1}, \zeta_{2}, \zeta_{3}\right)
$$

and that

$$
c>0, \quad K_{r s} \xi_{r} \xi_{s}>0 \text { for all non-zero vectors }\left(\xi_{1}, \xi_{2}, \xi_{3}\right)
$$

It follows from (54) that

$$
C_{r 3 s 3} \xi_{r} \xi_{s}>0 \text { for all non-zero vectors }\left(\xi_{1}, \xi_{2}, \xi_{3}\right)
$$


and

$$
C_{r \alpha s \beta} \xi_{r} \xi_{s} \zeta_{\alpha} \zeta_{\beta}>0 \text { for all non-zero vectors }\left(\xi_{1}, \xi_{2}, \xi_{3}\right),\left(\zeta_{1}, \zeta_{2}\right)
$$

We further assume that $\partial D$ is star shaped with respect to the origin so that $x_{\rho} n_{\rho} \geq h_{0}>0$, with $h_{0}>0$ constant. On this basis we observe that

$$
0 \leq \int_{\partial D\left(x_{3}\right)} x_{\rho} n_{\rho} C_{r \alpha m \beta} n_{\alpha} n_{\beta} \frac{\partial v_{r}}{\partial n} \frac{\partial \bar{v}_{m}}{\partial n} d s \leq d C \int_{\partial D\left(x_{3}\right)} \frac{\partial v_{r}}{\partial n} \frac{\partial \bar{v}_{r}}{\partial n} d s
$$

where

$$
\begin{aligned}
& C=\left(C_{r \alpha m \beta} C_{r \alpha m \beta}\right)^{1 / 2} \\
& d=\sup _{\left(x_{1}, x_{2}\right) \in \partial D}\left(x_{\alpha} x_{\alpha}\right)^{1 / 2}
\end{aligned}
$$

Similarly, we have

$$
0 \leq \int_{\partial D\left(x_{3}\right)} \frac{1}{\theta_{0}} x_{\rho} n_{\rho} K_{\alpha \beta} n_{\alpha} n_{\beta} \frac{\partial T}{\partial n} \frac{\partial \bar{T}}{\partial n} d s \leq \frac{d K}{\theta_{0}} \int_{\partial D\left(x_{3}\right)} \frac{\partial T}{\partial n} \frac{\partial \bar{T}}{\partial n} d s
$$

where

$$
K=\left(K_{\alpha \beta} K_{\alpha \beta}\right)^{1 / 2}
$$

Further, we introduce

$$
\begin{aligned}
& m_{0}=\max _{x_{3} \in[0, L]} \frac{\int_{\partial D\left(x_{3}\right)} \frac{\partial v_{r}}{\partial n} \frac{\partial \bar{v}_{r}}{\partial n} d s}{\int_{D\left(x_{3}\right)} v_{s} \bar{v}_{s} d a}, \quad \omega_{0}^{*}=\frac{1}{\rho} d C m_{0} \\
& m_{1}=\max _{x_{3} \in[0, L]} \frac{\int_{\partial D\left(x_{3}\right)} \frac{\partial T}{\partial n} \frac{\partial T}{\partial n} d s}{\int_{D\left(x_{3}\right)} T \bar{T} d a}, \quad \omega_{1}^{*}=\frac{1}{c} d K m_{1}
\end{aligned}
$$

and assume that

$$
\omega>\omega^{*}=\max \left(\omega_{0}^{*}, \omega_{1}^{*}\right)
$$
bounds

Whereas one cannot, in general, obtain $m_{0}$ and $m_{1}$ explicitly, we have the crude

$$
m_{0} \leq m_{0}^{*}, \quad m_{1} \leq m_{1}^{*}
$$

with

$$
m_{0}^{*}=\max _{v_{k} \in H_{0}^{1}(D)} \frac{\int_{\partial D\left(x_{3}\right)} \frac{\partial v_{r}}{\partial n} \frac{\partial \bar{v}_{r}}{\partial n} d s}{\int_{D\left(x_{3}\right)} v_{s} \bar{v}_{s} d a}, \quad m_{1}^{*}=\max _{T \in H_{0}^{1}(D)} \frac{\int_{\partial D\left(x_{3}\right)} \frac{\partial T}{\partial n} \frac{\partial \bar{T}}{\partial n} d s}{\int_{D\left(x_{3}\right)} T \bar{T} d a}
$$

So, when $m_{0}^{*}$ and $m_{1}^{*}$ are finite, we can take $\omega^{*}=\max \left(\frac{1}{\rho} d C m_{0}^{*}, \frac{1}{c} d K m_{1}^{*}\right)$ and so we obtain an explicit critical value for the frequency of vibration. 
Then the identity (53), relations (58), (61) and (65) give

$$
\begin{aligned}
& \frac{d}{d x_{3}} \int_{D\left(x_{3}\right)}\{\left(C_{3 s m n} v_{m, n}-i \omega M_{3 s} T\right)\left(\bar{v}_{s}+x_{\rho} \bar{v}_{s, \rho}\right) \\
&+\left(C_{3 s m n} \bar{v}_{m, n}+i \omega M_{3 s} \bar{T}\right)\left(v_{s}+x_{\rho} v_{s, \rho}\right)+\frac{1}{\theta_{0}} K_{33}\left(\bar{T} T_{, 3}+T \bar{T}_{, 3}\right) \\
&+\frac{x_{\alpha}}{\theta_{0}}\left[K_{3 \beta}\left(\bar{T}_{, \alpha} T_{, \beta}+T_{, \alpha} \bar{T}_{, \beta}\right)+K_{33}\left(\bar{T}_{, \alpha} T_{, 3}+T_{, \alpha} \bar{T}_{, 3}\right)\right] \\
&+x_{3}\left[\omega^{2}\left(\rho v_{s} \bar{v}_{s}+\frac{c}{\theta_{0}} T \bar{T}\right)+C_{r 3 m 3} v_{r, 3} \bar{v}_{m, 3}+\frac{1}{\theta_{0}} K_{33} T_{, 3} \bar{T}_{, 3}\right. \\
&\left.\left.-\left(C_{r \alpha m \beta} v_{r, \alpha} \bar{v}_{m, \beta}+\frac{1}{\theta_{0}} K_{\alpha, \beta} T_{, \alpha} \bar{T}_{, \beta}\right)+i \omega M_{r \alpha}\left(T \bar{v}_{r, \alpha}-\bar{T} v_{r, \alpha}\right)\right]\right\} d a \\
& \geq \int_{D\left(x_{3}\right)}\left(C_{r s m n} v_{r, s} \bar{v}_{m, n}+\frac{1}{\theta_{0}} K_{r s} T_{, r} \bar{T}_{, s}\right) d a
\end{aligned}
$$

Our objective now is to find measures that are able to furnish information on the spatial evolution of the amplitude $\left(v_{r}, T\right)$ for the entire class of anisotropic strongly elliptic thermoelastic materials.

We now pursue our method for rhombic systems.

\section{SPATIAL EVOLUTION IN THE HIGH FREQUENCY RANGE FOR RHOMBIC MATERIALS}

Suppose the cylinder is filled by a rhombic thermoelastic material with the group $\mathscr{C}_{3}$ generated by $\mathbf{R}_{e_{3}}^{\pi}, \mathbf{R}_{e_{2}}^{\pi}$ (here $\mathbf{R}_{e}^{\alpha}$ is the orthogonal tensor corresponding to a right-handed rotation through the angle $\alpha \in(0,2 \pi)$, about an axis in the direction of the unit vector $\mathbf{e})$. For such a class of materials we have

$$
\begin{aligned}
C_{1123} & =C_{1131}=C_{1112}=C_{2223}=C_{2231}=C_{2212}=0 \\
C_{3323} & =C_{3331}=C_{3312}=C_{2331}=C_{2312}=C_{3112}=0 \\
K_{3 \alpha} & =K_{\alpha 3}=0, \quad M_{3 \alpha}=M_{\alpha 3}=0
\end{aligned}
$$

Furthermore, we set

$$
\begin{aligned}
& c_{11}=C_{1111}, \quad c_{22}=C_{2222}, \quad c_{33}=C_{3333}, \quad c_{12}=C_{1122}, \quad c_{23}=C_{2233} \\
& c_{13}=C_{1133}, \quad c_{44}=C_{2323}, \quad c_{55}=C_{1313}, \quad c_{66}=C_{1212}
\end{aligned}
$$

Then the necessary and sufficient conditions for strong ellipticity of the elasticity tensor for a rhombic material are [23]

$$
\begin{aligned}
c_{11} & >0, \quad c_{22}>0, \quad c_{33}>0, \quad c_{44}>0, \quad c_{55}>0, \quad c_{66}>0 \\
& -2 c_{66}+\varkappa_{3}^{i} \sqrt{c_{11} c_{22}}<c_{12}<\varkappa_{3}^{s} \sqrt{c_{11} c_{22}} \\
& -2 c_{44}+\varkappa_{1}^{i} \sqrt{c_{22} c_{33}}<c_{23}<\varkappa_{1}^{s} \sqrt{c_{22} c_{33}} \\
& -2 c_{55}+\varkappa_{2}^{i} \sqrt{c_{11} c_{33}}<c_{13}<\varkappa_{2}^{s} \sqrt{c_{11} c_{33}}
\end{aligned}
$$


where $\left(\varkappa_{1}^{i}, \varkappa_{1}^{s}\right),\left(\varkappa_{2}^{i}, \varkappa_{2}^{s}\right)$ and $\left(\varkappa_{3}^{i}, \varkappa_{3}^{s}\right)$ are solutions with respect to $x, y$ and $z$ of the equation $x^{2}+y^{2}+z^{2}-2 x y z-1=0$, satisfying $|x|<1,|y|<1,|z|<1$ and $x \in\left\{\frac{c_{23}}{\sqrt{c_{22} c_{33}}}, \frac{c_{23}+2 c_{44}}{\sqrt{c_{22} c_{33}}}\right\}, y \in\left\{\frac{c_{13}}{\sqrt{c_{11} c_{33}}}, \frac{c_{13}+2 c_{55}}{\sqrt{c_{11} c_{33}}}\right\}, \quad z \in\left\{\frac{c_{12}}{\sqrt{c_{11} c_{22}}}, \frac{c_{12}+2 c_{66}}{\sqrt{c_{11} c_{22}}}\right\}$. This statement expressed by (72) is equivalent with the fact that all points $P(x, y, z)$, with coordinates $x \in\left\{\frac{c_{23}}{\sqrt{c_{22} c_{33}}}, \frac{c_{23}+2 c_{44}}{\sqrt{c_{22} c_{33}}}\right\}, y \in\left\{\frac{c_{13}}{\sqrt{c_{11} c_{33}}}, \frac{c_{13}+2 c_{55}}{\sqrt{c_{11} c_{33}}}\right\}, z \in\left\{\frac{c_{12}}{\sqrt{c_{11} c_{22}}}, \frac{c_{12}+2 c_{66}}{\sqrt{c_{11} c_{22}}}\right\}$, lie inside of the region limited by the surface $S(x, y, z) \equiv x^{2}+y^{2}+z^{2}-2 x y z-1=0$, with $|x|<1,|y|<1,|z|<1$.

For rhombic materials the relation (68) gives

$$
\begin{aligned}
& \frac{d}{d x_{3}} \int_{D\left(x_{3}\right)}\left\{c_{55}\left(v_{1,3}+v_{3,1}\right)\left(\bar{v}_{1}+x_{\rho} \bar{v}_{1, \rho}\right)+c_{55}\left(\bar{v}_{1,3}+\bar{v}_{3,1}\right)\left(v_{1}+x_{\rho} v_{1, \rho}\right)\right. \\
& +c_{44}\left(v_{2,3}+v_{3,2}\right)\left(\bar{v}_{2}+x_{\rho} \bar{v}_{2, \rho}\right)+c_{44}\left(\bar{v}_{2,3}+\bar{v}_{3,2}\right)\left(v_{2}+x_{\rho} v_{2, \rho}\right) \\
& +\left(c_{13} v_{1,1}+c_{23} v_{2,2}+c_{33} v_{3,3}\right)\left(\bar{v}_{3}+x_{\rho} \bar{v}_{3, \rho}\right) \\
& +\left(c_{13} \bar{v}_{1,1}+c_{23} \bar{v}_{2,2}+c_{33} \bar{v}_{3,3}\right)\left(v_{3}+x_{\rho} v_{3, \rho}\right) \\
& +i \omega M_{33}\left[\bar{T}\left(v_{3}+x_{\rho} v_{3, \rho}\right)-T\left(\bar{v}_{3}+x_{\rho} \bar{v}_{3, \rho}\right)\right] \\
& +\frac{1}{\theta_{0}} K_{33}\left(\bar{T} T_{, 3}+T \bar{T}_{, 3}\right)+\frac{x_{\alpha}}{\theta_{0}} K_{33}\left(\bar{T}_{, \alpha} T_{, 3}+T_{, \alpha} \bar{T}_{, 3}\right) \\
& +x_{3}\left[c_{55} v_{1,3} \bar{v}_{1,3}+c_{44} v_{2,3} \bar{v}_{2,3}+c_{33} v_{3,3} \bar{v}_{3,3}-c_{66}\left(v_{1,2}+v_{2,1}\right)\left(\bar{v}_{1,2}+\bar{v}_{2,1}\right)\right. \\
& -\left(c_{11} v_{1,1} \bar{v}_{1,1}+c_{22} v_{2,2} \bar{v}_{2,2}+c_{12}\left(v_{1,1} \bar{v}_{2,2}+\bar{v}_{1,1} v_{2,2}\right)+c_{55} v_{3,1} \bar{v}_{3,1}\right. \\
& \left.+c_{44} v_{3,2} \bar{v}_{3,2}\right)+i \omega M_{\alpha \beta}\left(T \bar{v}_{\alpha, \beta}-\bar{T} v_{\alpha, \beta}\right) \\
& \left.\left.+\frac{1}{\theta_{0}}\left(K_{33} T_{, 3} \bar{T}_{, 3}-K_{\alpha \beta} T_{, \alpha} \bar{T}_{, \beta}\right)+\omega^{2}\left(\rho v_{s} \bar{v}_{s}+\frac{c}{\theta_{0}} T \bar{T}\right)\right]\right\} d a \\
& \geq \int_{D\left(x_{3}\right)}\left[c_{11} v_{1,1} \bar{v}_{1,1}+c_{22} v_{2,2} \bar{v}_{2,2}+c_{33} v_{3,3} \bar{v}_{3,3}+c_{12}\left(v_{1,1} \bar{v}_{2,2}+\bar{v}_{1,1} v_{2,2}\right)\right. \\
& \left.+c_{23}\left(v_{2,2} \bar{v}_{3,3}+\bar{v}_{2,2} v_{3,3}\right)+c_{13}\left(v_{1,1} \bar{v}_{3,3}+\bar{v}_{1,1} v_{3,3}\right)\right] d a \\
& +\int_{D\left(x_{3}\right)}\left[c_{66} v_{1,2} \bar{v}_{1,2}+c_{66}\left(v_{1,2} \bar{v}_{2,1}+\bar{v}_{1,2} v_{2,1}\right)+c_{66} v_{2,1} \bar{v}_{2,1}\right] d a \\
& +\int_{D\left(x_{3}\right)}\left[c_{55} v_{1,3} \bar{v}_{1,3}+c_{55}\left(v_{1,3} \bar{v}_{3,1}+\bar{v}_{1,3} v_{3,1}\right)+c_{55} v_{3,1} \bar{v}_{3,1}\right] d a \\
& +\int_{D\left(x_{3}\right)}\left[c_{44} v_{2,3} \bar{v}_{2,3}+c_{44}\left(v_{2,3} \bar{v}_{3,2}+\bar{v}_{2,3} v_{3,2}\right)+c_{44} v_{3,2} \bar{v}_{3,2}\right] d a \\
& +\int_{D\left(x_{3}\right)}\left(\frac{1}{\theta_{0}} K_{\alpha \beta} T_{, \alpha} \bar{T}_{, \beta}+\frac{1}{\theta_{0}} K_{33} T_{, 3} \bar{T}_{, 3}\right) d a
\end{aligned}
$$

On the other hand, by means of the lateral boundary condition in (16) we have the following identities

$$
\begin{aligned}
\frac{d}{d x_{3}} \int_{D\left(x_{3}\right)}\left(v_{1,1} \bar{v}_{3}+\bar{v}_{1,1} v_{3}\right) d a & =-\frac{d}{d x_{3}} \int_{D\left(x_{3}\right)}\left(v_{1} \bar{v}_{3,1}+\bar{v}_{1} v_{3,1}\right) d a \\
& =\int_{D\left(x_{3}\right)}\left(v_{1,1} \bar{v}_{3,3}+\bar{v}_{1,1} v_{3,3}\right) d a-\int_{D\left(x_{3}\right)}\left(v_{1,3} \bar{v}_{3,1}+\bar{v}_{1,3} v_{3,1}\right) d a
\end{aligned}
$$




$$
\begin{aligned}
\frac{d}{d x_{3}} \int_{D\left(x_{3}\right)}\left(v_{2,2} \bar{v}_{3}+\bar{v}_{2,2} v_{3}\right) d a & =-\frac{d}{d x_{3}} \int_{D\left(x_{3}\right)}\left(v_{2} \bar{v}_{3,2}+\bar{v}_{2} v_{3,2}\right) d a \\
& =\int_{D\left(x_{3}\right)}\left(v_{2,2} \bar{v}_{3,3}+\bar{v}_{2,2} v_{3,3}\right) d a-\int_{D\left(x_{3}\right)}\left(v_{2,3} \bar{v}_{3,2}+\bar{v}_{2,3} v_{3,2}\right) d a \\
0 & =\int_{D\left(x_{3}\right)}\left(v_{1,1} \bar{v}_{2,2}+\bar{v}_{1,1} v_{2,2}\right) d a-\int_{D\left(x_{3}\right)}\left(v_{1,2} \bar{v}_{3,1}+\bar{v}_{1,2} v_{2,1}\right) d a
\end{aligned}
$$

Therefore, by introducing the function

$$
\begin{aligned}
I\left(x_{3}\right)=-\int_{D\left(x_{3}\right)}\{[ & \left.c_{55} v_{1,3}+\left(c_{55}-\kappa_{2}\right) v_{3,1}\right]\left(\bar{v}_{1}+x_{\rho} \bar{v}_{1, \rho}\right) \\
& +\left[c_{55} \bar{v}_{1,3}+\left(c_{55}-\kappa_{2}\right) \bar{v}_{3,1}\right]\left(v_{1}+x_{\rho} v_{1, \rho}\right) \\
& +\left[c_{44} v_{2,3}+\left(c_{44}-\kappa_{1}\right) v_{3,2}\right]\left(\bar{v}_{2}+x_{\rho} \bar{v}_{2, \rho}\right) \\
& +\left[c_{44} \bar{v}_{2,3}+\left(c_{44}-\kappa_{1}\right) \bar{v}_{3,2}\right]\left(v_{2}+x_{\rho} v_{2, \rho}\right) \\
+ & +\left[\left(c_{13}+\kappa_{2}\right) v_{1,1}+\left(c_{23}+\kappa_{1}\right) v_{2,2}+c_{33} v_{3,3}\right]\left(\bar{v}_{3}+x_{\rho} \bar{v}_{3, \rho}\right) \\
+ & {\left[\left(c_{13}+\kappa_{2}\right) \bar{v}_{1,1}+\left(c_{23}+\kappa_{1}\right) \bar{v}_{2,2}+c_{33} \bar{v}_{3,3}\right]\left(v_{3}+x_{\rho} v_{3, \rho}\right) } \\
+ & i \omega M_{33}\left[\bar{T}\left(v_{3}+x_{\rho} v_{3, \rho}\right)-T\left(\bar{v}_{3}+x_{\rho} \bar{v}_{3, \rho}\right)\right] \\
+ & \frac{1}{\theta_{0}} K_{33}\left(\bar{T} T_{, 3}+T \bar{T}_{, 3}\right)+\frac{x_{\alpha}}{\theta_{0}} K_{33}\left(\bar{T}_{, \alpha} T_{, 3}+T_{, \alpha} \bar{T}_{, 3}\right) \\
+ & x_{3}\left[c_{55} v_{1,3} \bar{v}_{1,3}+c_{44} v_{2,3} \bar{v}_{2,3}+c_{33} v_{3,3} \bar{v}_{3,3}-c_{66}\left(v_{1,2}+v_{2,1}\right)\left(\bar{v}_{1,2}+\bar{v}_{2,1}\right)\right. \\
& \quad-\left(c_{11} v_{1,1} \bar{v}_{1,1}+c_{22} v_{2,2} \bar{v}_{2,2}+c_{12}\left(v_{1,1} \bar{v}_{2,2}+\bar{v}_{1,1} v_{2,2}\right)+c_{55} v_{3,1} \bar{v}_{3,1}\right. \\
& \left.\quad+c_{44} v_{3,2} \bar{v}_{3,2}\right)+i \omega M_{\alpha \beta}\left(T \bar{v}_{\alpha, \beta}-\bar{T} v_{\alpha, \beta}\right) \\
& \left.\left.+\frac{1}{\theta_{0}}\left(K_{33} T_{, 3} \bar{T}_{, 3}-K_{\alpha \beta} T_{, \alpha} \bar{T}_{, \beta}\right)+\omega^{2}\left(\rho v_{s} \bar{v}_{s}+\frac{c}{\theta_{0}} T \bar{T}\right)\right]\right\} d a(75)
\end{aligned}
$$

the relations (73) and (74) give

$$
\begin{aligned}
-\frac{d I}{d x_{3}}\left(x_{3}\right) \geq & \int_{D\left(x_{3}\right)}\left[c_{11} v_{1,1} \bar{v}_{1,1}+c_{22} v_{2,2} \bar{v}_{2,2}+c_{33} v_{3,3} \bar{v}_{3,3}+\left(c_{12}+\kappa_{3}\right)\left(v_{1,1} \bar{v}_{2,2}+\bar{v}_{1,1} v_{2,2}\right)\right. \\
& \left.\quad+\left(c_{23}+\kappa_{1}\right)\left(v_{2,2} \bar{v}_{3,3}+\bar{v}_{2,2} v_{3,3}\right)+\left(c_{13}+\kappa_{2}\right)\left(v_{1,1} \bar{v}_{3,3}+\bar{v}_{1,1} v_{3,3}\right)\right] d a \\
& +\int_{D\left(x_{3}\right)}\left[c_{66} v_{1,2} \bar{v}_{1,2}+\left(c_{66}-\kappa_{3}\right)\left(v_{1,2} \bar{v}_{2,1}+\bar{v}_{1,2} v_{2,1}\right)+c_{66} v_{2,1} \bar{v}_{2,1}\right] d a \\
& +\int_{D\left(x_{3}\right)}\left[c_{55} v_{1,3} \bar{v}_{1,3}+\left(c_{55}-\kappa_{2}\right)\left(v_{1,3} \bar{v}_{3,1}+\bar{v}_{1,3} v_{3,1}\right)+c_{55} v_{3,1} \bar{v}_{3,1}\right] d a \\
& +\int_{D\left(x_{3}\right)}\left[c_{44} v_{2,3} \bar{v}_{2,3}+\left(c_{44}-\kappa_{1}\right)\left(v_{2,3} \bar{v}_{3,2}+\bar{v}_{2,3} v_{3,2}\right)+c_{44} v_{3,2} \bar{v}_{3,2}\right] d a \\
& +\int_{D\left(x_{3}\right)}\left(\frac{1}{\theta_{0}} K_{\alpha \beta} T_{, \alpha} \bar{T}_{, \beta}+\frac{1}{\theta_{0}} K_{33} T_{, 3} \bar{T}_{3,3}\right) d a
\end{aligned}
$$


where $\varkappa_{1}, \varkappa_{2}$ and $\varkappa_{3}$ are positive parameters at our disposal. Further, in view of the assumptions (71) and (72) we can choose $\varkappa_{1} \in\left[0,2 c_{44}\right], \varkappa_{2} \in\left[0,2 c_{55}\right], \varkappa_{3} \in\left[0,2 c_{66}\right]$ so that $P(x, y, z)$, with coordinates $x=\frac{c_{23}+\varkappa_{1}}{\sqrt{c_{22} c_{33}}}, y=\frac{c_{13}+\varkappa_{2}}{\sqrt{c_{11} c_{33}}}, z=\frac{c_{12}+\varkappa_{3}}{\sqrt{c_{11} c_{22}}}$, lies inside the region limited by the surface $S(x, y, z)$. With these choices we have

$$
\begin{gathered}
c_{44}\left(v_{2,3} \bar{v}_{2,3}+v_{3,2} \bar{v}_{3,2}\right)+\left(c_{44}-\varkappa_{1}\right)\left(v_{2,3} \bar{v}_{3,2}+\bar{v}_{2,3} v_{3,2}\right) \geq \xi_{1}\left(v_{2,3} \bar{v}_{2,3}+v_{3,2} \bar{v}_{3,2}\right) \\
c_{55}\left(v_{1,3} \bar{v}_{1,3}+v_{3,1} \bar{v}_{3,1}\right)+\left(c_{55}-\varkappa_{2}\right)\left(v_{1,3} \bar{v}_{3,1}+\bar{v}_{1,3} v_{3,1}\right) \geq \xi_{2}\left(v_{1,3} \bar{v}_{1,3}+v_{3,1} \bar{v}_{3,1}\right) \\
c_{66}\left(v_{1,2} \bar{v}_{1,2}+v_{2,1} \bar{v}_{2,1}\right)+\left(c_{66}-\varkappa_{3}\right)\left(v_{1,2} \bar{v}_{2,1}+\bar{v}_{1,2} v_{2,1}\right) \geq \xi_{3}\left(v_{1,2} \bar{v}_{1,2}+v_{2,1} \bar{v}_{2,1}\right) \\
c_{11} v_{1,1} \bar{v}_{1,1}+c_{22} v_{2,2} \bar{v}_{2,2}+c_{33} v_{3,3} \bar{v}_{3,3}+\left(c_{12}+\varkappa_{3}\right)\left(v_{1,1} \bar{v}_{2,2}+\bar{v}_{1,1} v_{2,2}\right) \\
+c_{13}\left(v_{1,1} \bar{v}_{3,3}+\bar{v}_{1,1} v_{3,3}\right)+c_{23}\left(v_{2,2} \bar{v}_{3,3}+\bar{v}_{2,2} v_{3,3}\right) \\
\geq \xi_{4}\left(v_{1,1} \bar{v}_{1,1}+v_{2,2} \bar{v}_{2,2}+v_{3,3} \bar{v}_{3,3}\right) \\
K_{\alpha \beta} T_{, \alpha} \bar{T}_{, \beta} \geq k_{0} T_{, \alpha} \bar{T}_{, \alpha}
\end{gathered}
$$

where

$$
\xi_{1}=\min \left(2 c_{44}-\varkappa_{1}, \varkappa_{1}\right), \quad \xi_{2}=\min \left(2 c_{55}-\varkappa_{2}, \varkappa_{2}\right), \quad \xi_{3}=\min \left(2 c_{66}-\varkappa_{3}, \varkappa_{3}\right)
$$

$\xi_{4}$ is the lowest positive eigenvalue of the linear transformation given by the $3 \times 3$ matrix

$$
\left(\begin{array}{ccc}
c_{11} & c_{12}+\varkappa_{3} & c_{13}+\varkappa_{2} \\
c_{12}+\varkappa_{3} & c_{22} & c_{23}+\varkappa_{1} \\
c_{13}+\varkappa_{2} & c_{23}+\varkappa_{1} & c_{33}
\end{array}\right)
$$

and $k_{0}$ is the lowest positive eigenvalue of $K_{\alpha \beta}$. On this basis we have

$$
\begin{aligned}
-\frac{d I}{d x_{3}}\left(x_{3}\right) \geq & \int_{D\left(x_{3}\right)} \xi_{4}\left(v_{1,1} \bar{v}_{1,1}+v_{2,2} \bar{v}_{2,2}+v_{3,3} \bar{v}_{3,3}\right) d a+\int_{D\left(x_{3}\right)} \xi_{3}\left(v_{1,2} \bar{v}_{1,2}+v_{2,1} \bar{v}_{2,1}\right) d a \\
& +\int_{D\left(x_{3}\right)} \xi_{2}\left(v_{1,3} \bar{v}_{1,3}+v_{3,1} \bar{v}_{3,1}\right) d a+\int_{D\left(x_{3}\right)} \xi_{1}\left(v_{2,3} \bar{v}_{2,3}+v_{3,2} \bar{v}_{3,2}\right) d a \\
& +\int_{D\left(x_{3}\right)}\left(\frac{k_{0}}{\theta_{0}} T_{, \alpha} \bar{T}_{, \beta}+\frac{1}{\theta_{0}} K_{33} T_{, 3} \bar{T}_{, 3}\right) d a \geq 0
\end{aligned}
$$

and hence $I\left(x_{3}\right)$ is a non-increasing function with respect to $x_{3}$ on $[0, L]$.

On the other hand, in view of the lateral boundary condition in (16) we have

$$
\begin{aligned}
\int_{D\left(x_{3}\right)} v_{1, \alpha} \bar{v}_{1, \alpha} d a & \geq \lambda \int_{D\left(x_{3}\right)} v_{1} \bar{v}_{1} d a, \quad \int_{D\left(x_{3}\right)} v_{2, \alpha} \bar{v}_{2, \alpha} d a \geq \lambda \int_{D\left(x_{3}\right)} v_{2} \bar{v}_{2} d a \\
\int_{D\left(x_{3}\right)} v_{3, \alpha} \bar{v}_{3, \alpha} d a & \geq \lambda \int_{D\left(x_{3}\right)} v_{3} \bar{v}_{3} d a \\
\int_{D\left(x_{3}\right)} T_{, \alpha} \bar{T}_{, \alpha} d a & \geq \lambda \int_{D\left(x_{3}\right)} T \bar{T} d a
\end{aligned}
$$


where $\lambda>0$ is the first eigenvalue in the two-dimensional clamped membrane eigenvalue problem for the cross section $D\left(x_{3}\right)$. Further, in view of our choice we have $\left|c_{55}-\varkappa_{2}\right|<c_{55},\left|c_{44}-\varkappa_{1}\right|<c_{44},\left|c_{13}+\varkappa_{2}\right|<\sqrt{c_{11} c_{33}}$ and $\left|c_{23}+\varkappa_{1}\right|<\sqrt{c_{22} c_{33}}$. Then, by means of the Schwarz and arithmetic-geometric inequalities and by using the inequalities (85) and (86), we obtain

$$
\begin{aligned}
&\left|I\left(x_{3}\right)\right| \leq \int_{D\left(x_{3}\right)}\{ N_{1} v_{1,1} \bar{v}_{1,1}+ \\
&+\left.N_{2} v_{2,2} \bar{v}_{2,2}+N_{3} v_{3,3} \bar{v}_{3,3}+N_{2,3} \bar{v}_{2,3}+N_{8,2} \bar{v}_{1,2}+N_{3,1} \bar{v}_{3,1}+N_{9} v_{3,2} \bar{v}_{3,2}+N_{10} T_{, \alpha} \bar{T}_{, \alpha}+N_{11} T_{, 3} \bar{T}_{1,3}\right\} d a \\
&+x_{3} \int_{D\left(x_{3}\right)}\left\{N_{1}^{\prime} v_{1,1} \bar{v}_{1,1}+N_{2}^{\prime} v_{2,2} \bar{v}_{2,2}+N_{3}^{\prime} v_{3,3} \bar{v}_{3,3}+N_{4}^{\prime} v_{1,2} \bar{v}_{1,2}+N_{5}^{\prime} v_{2,1} \bar{v}_{2,1}\right. \\
&+N_{6}^{\prime} v_{1,3} \bar{v}_{1,3}+N_{7}^{\prime} v_{2,3} \bar{v}_{2,3}+N_{8}^{\prime} v_{3,1} \bar{v}_{3,1}+N_{9}^{\prime} v_{3,2} \bar{v}_{3,2} \\
&\left.+N_{10}^{\prime} T_{, \alpha} \bar{T}_{, \alpha}+N_{11}^{\prime} T_{, 3} \bar{T}_{, 3}\right\} d a
\end{aligned}
$$

where

$$
\begin{aligned}
& N_{1}=\frac{1}{\sqrt{\lambda}}\left[2 c_{55}\left(1+\lambda d^{2}\right)+\sqrt{c_{11} c_{33}}\right], \quad N_{2}=\frac{1}{\sqrt{\lambda}}\left(2 c_{44}\left(1+\lambda d^{2}\right)+\sqrt{c_{22} c_{33}}\right) \\
& N_{3}=\frac{c_{33}}{\sqrt{\lambda}}, \quad N_{4}=\frac{2 c_{55}}{\sqrt{\lambda}}\left(1+\lambda d^{2}\right), \quad N_{5}=\frac{2 c_{44}}{\sqrt{\lambda}}\left(1+\lambda d^{2}\right), \quad N_{6}=\frac{2 c_{55}}{\sqrt{\lambda}}, \quad N_{7}=\frac{2 c_{44}}{\sqrt{\lambda}} \\
& N_{8}=\frac{2}{\sqrt{\lambda}}\left[c_{55}+\left(1+\lambda d^{2}\right)\left(\sqrt{c_{11} c_{33}}+\sqrt{c_{22} c_{33}}+c_{33}+\frac{\omega}{\sqrt{\lambda}}\left|M_{33}\right| \sqrt{\frac{\theta_{0} c_{33}}{k_{0}}}\right)\right] \\
& N_{9}=\frac{2}{\sqrt{\lambda}}\left[c_{44}+\left(1+\lambda d^{2}\right)\left(\sqrt{c_{11} c_{33}}+\sqrt{c_{22} c_{33}}+c_{33}+\frac{\omega}{\sqrt{\lambda}}\left|M_{33}\right| \sqrt{\frac{\theta_{0} c_{33}}{k_{0}}}\right)\right] \\
& N_{10}=\frac{\omega}{\lambda}\left|M_{33}\right| \sqrt{\frac{k_{0}}{\theta_{0} c_{33}}}+\frac{2}{\theta_{0}} \sqrt{\frac{K_{33} k_{0}}{\lambda}}\left(1+\lambda d^{2}\right), \quad N_{11}=\frac{1}{\theta_{0}} \sqrt{\frac{K_{33}}{\lambda k_{0}}} K_{33}
\end{aligned}
$$

and

$$
\begin{aligned}
& N_{1}^{\prime}=c_{11}+\left|c_{12}\right|+\omega \sqrt{\frac{\theta_{0} c_{11}}{\lambda k_{0}} M_{\alpha \beta} M \alpha \beta}+\frac{\omega^{2} \rho}{\lambda}, \quad N_{2}^{\prime}=c_{22}-c_{11}+N_{1}^{\prime}, \quad N_{3}^{\prime}=c_{33} \\
& N_{4}^{\prime}=N_{5}^{\prime}=2 c_{66}+\omega \sqrt{\frac{\theta_{0} c_{11}}{\lambda k_{0}} M_{\alpha \beta} M \alpha \beta}+\frac{\omega^{2} \rho}{\lambda}, \quad N_{6}^{\prime}=c_{55}, \quad N_{7}^{\prime}=c_{44}, \quad N_{8}^{\prime}=c_{55}+\frac{\omega^{2} \rho}{\lambda} \\
& N_{9}^{\prime}=c_{44}+\frac{\omega^{2} \rho}{\lambda}, \quad N_{10}^{\prime}=\frac{K}{\theta_{0}}+\omega \sqrt{\frac{k_{0}}{\lambda c_{11}} M_{\alpha \beta} M_{\alpha \beta}}+\frac{\omega^{2} c}{\lambda \theta_{0}}, \quad N_{11}^{\prime}=\frac{1}{\theta_{0}} K_{33}
\end{aligned}
$$

and $K$ is the maximum eigenvalue of $K_{\alpha \beta}$. Therefore, if we use the estimate (87) in combination with relation (84), then we obtain the following differential inequality

$$
\left|I\left(x_{3}\right)\right| \leq-\left(\alpha+\beta x_{3}\right) \frac{d I}{d x_{3}}\left(x_{3}\right) \text { for all } x_{3} \in[0, L]
$$


where

$$
\begin{aligned}
& \alpha=\max \left\{\frac{1}{\xi_{4}} \max \left(N_{1}, N_{2}, N_{3}\right), \frac{1}{\xi_{3}} \max \left(N_{4}, N_{5}\right), \frac{1}{\xi_{2}} \max \left(N_{6}, N_{8}\right),\right.\left.\frac{1}{\xi_{1}} \max \left(N_{7}, N_{9}\right) \frac{\theta_{0} N_{10}}{k_{0}}, \frac{\theta_{0} N_{11}}{K_{33}}\right\}, \\
& \beta=\max \left\{\frac{1}{\xi_{4}} \max \left(N_{1}^{\prime}, N_{2}^{\prime}, N_{3}^{\prime}\right), \frac{1}{\xi_{3}} \max \left(N_{4}^{\prime}, N_{5}^{\prime}\right), \frac{1}{\xi_{2}} \max \left(N_{6}^{\prime}, N_{8}^{\prime}\right),\right. \\
&\left.\frac{1}{\xi_{1}} \max \left(N_{7}^{\prime}, N_{9}^{\prime}\right) \frac{\theta_{0} N_{10}^{\prime}}{k_{0}}, \frac{\theta_{0} N_{11}^{\prime}}{K_{33}}\right\}
\end{aligned}
$$

To discuss the implications of the differential inequality (90), we suppose first that $I\left(x_{3}^{*}\right) \leq 0$ for some fixed $x_{3}^{*} \in[0, L]$. Because $I\left(x_{3}\right)$ is non-increasing with respect to $x_{3}$, we have $0 \geq I\left(x_{3}^{*}\right) \geq I\left(x_{3}\right)$ for $x_{3}^{*} \leq x_{3} \leq L$, and accordingly we can conclude from (90)) that

$$
\frac{d I}{d x_{3}}\left(x_{3}\right)-\frac{1}{\alpha+\beta x_{3}} I\left(x_{3}\right) \leq 0, \quad x_{3}^{*} \leq x_{3} \leq L
$$

which after integration leads to the growth estimate

$$
-I\left(x_{3}\right) \geq-I\left(x_{3}^{*}\right)\left(\frac{\alpha+\beta x_{3}}{\alpha+\beta x_{3}^{*}}\right)^{\frac{1}{\beta}}, \quad x_{3}^{*} \leq x_{3} \leq L
$$

Considering the case of a semi-infinite cylinder, we can conclude from (94) that $I\left(x_{3}\right)$, supposed to be not identically zero, becomes unbounded for assymptotically large values of $x_{3}$, which by means of relations (53) and (75) implies the unboundedness as $x_{3} \rightarrow \infty$ of the energetic measure

$$
\begin{aligned}
\mathscr{E}\left(x_{3}^{*}, x_{3}\right)= & \int_{x_{3}^{*}}^{x_{3}} d \xi \int_{D(\xi)}\left[c_{11} v_{1,1} \bar{v}_{1,1}+c_{22} v_{2,2} \bar{v}_{2,2}+c_{33} v_{3,3} \bar{v}_{3,3}+\left(c_{12}+\kappa_{3}\right)\left(v_{1,1} \bar{v}_{2,2}+\bar{v}_{1,1} v_{2,2}\right)\right. \\
& \left.+\left(c_{23}+\kappa_{1}\right)\left(v_{2,2} \bar{v}_{3,3}+\bar{v}_{2,2} v_{3,3}\right)+\left(c_{13}+\kappa_{2}\right)\left(v_{1,1} \bar{v}_{3,3}+\bar{v}_{1,1} v_{3,3}\right)\right] d a \\
& +\int_{x_{3}^{*}}^{x_{3}} d \xi \int_{D(\xi)}\left[c_{66} v_{1,2} \bar{v}_{1,2}+\left(c_{66}-\kappa_{3}\right)\left(v_{1,2} \bar{v}_{2,1}+\bar{v}_{1,2} v_{2,1}\right)+c_{66} v_{2,1} \bar{v}_{2,1}\right] d a \\
& +\int_{x_{3}^{*}}^{x_{3}} d \xi \int_{D(\xi)}\left[c_{55} v_{1,3} \bar{v}_{1,3}+\left(c_{55}-\kappa_{2}\right)\left(v_{1,3} \bar{v}_{3,1}+\bar{v}_{1,3} v_{3,1}\right)+c_{55} v_{3,1} \bar{v}_{3,1}\right] d a \\
& +\int_{x_{3}^{*}}^{x_{3}} d \xi \int_{D(\xi)}\left[c_{44} v_{2,3} \bar{v}_{2,3}+\left(c_{44}-\kappa_{1}\right)\left(v_{2,3} \bar{v}_{3,2}+\bar{v}_{2,3} v_{3,2}\right)+c_{44} v_{3,2} \bar{v}_{3,2}\right] d a \\
& +\int_{x_{3}^{*}}^{x_{3}} d \xi \int_{D(\xi)} \omega^{2}\left(\rho v_{s} \bar{v}_{s}+\frac{c}{\theta_{0}} T \bar{T}\right) d a-\int_{x_{3}^{*}}^{x_{3}} d \xi \int_{\partial D(\xi)} x_{\rho} n_{\rho} C_{r \alpha m \beta} n_{\alpha} n_{\beta} \frac{\partial v_{r}}{\partial n} \frac{\partial \bar{v}_{m}}{\partial n} d s \\
& -\int_{x_{3}^{*}}^{x_{3}} d \xi \int_{\partial D(\xi)} \frac{1}{\theta_{0}} x_{\rho} n_{\rho} K_{\alpha \beta} n_{\alpha} n_{\beta} \frac{\partial T}{\partial n} \frac{\partial T}{\partial n} d s \\
& +\int_{x_{3}^{*}}^{x_{3}} d \xi \int_{D(\xi)}\left(\frac{1}{\theta_{0}} K_{\alpha \beta} T_{, \alpha} \bar{T}_{, \beta}+\frac{1}{\theta_{0}} K_{33} T_{, 3} \bar{T}_{, 3}\right) d a
\end{aligned}
$$


We next suppose that $I\left(x_{3}\right)>0$ for all $x_{3} \in[0, L]$. Then the appropriate component inequality of (90) gives

$$
\frac{d I}{d x_{3}}\left(x_{3}\right)+\frac{1}{\alpha+\beta x_{3}} I\left(x_{3}\right) \leq 0, \quad 0 \leq x_{3} \leq L
$$

which after integration provides the alternative decay estimate

$$
0<I\left(x_{3}\right) \leq I(0)\left(1+\frac{\beta}{\alpha} x_{3}\right)^{-\frac{1}{\beta}}, \quad 0 \leq x_{3} \leq L
$$

We observe for the semi-infinite cylinder that the relations (53) and (97) imply that $\lim _{x_{3} \rightarrow \infty} \mathscr{E}\left(x_{3}^{*}, x_{3}\right)=\mathscr{E}\left(x_{3}^{*}, \infty\right)$ exists for all $x_{3}^{*} \geq 0$ and is finite and, moreover, it satisfies the following decay estimate

$$
0 \leq \mathscr{E}\left(x_{3}, \infty\right) \leq I(0)\left(1+\frac{\beta}{\alpha} x_{3}\right)^{-\frac{1}{\beta}}, \quad 0 \leq x_{3} \leq L
$$

\section{CONCLUDING REMARKS}

It may be remarked that the first order differential inequality (90) is different from those common used for establishing exponential spatial estimates of the Saint Venant type and this is due to the appearance of the constant $\beta$ that depends on the estimate of the term

$$
\begin{gathered}
\mathscr{F}=\int_{D\left(x_{3}\right)}\left[c_{55} v_{1,3} \bar{v}_{1,3}+c_{44} v_{2,3} \bar{v}_{2,3}+c_{33} v_{3,3} \bar{v}_{3,3}-c_{66}\left(v_{1,2}+v_{2,1}\right)\left(\bar{v}_{1,2}+\bar{v}_{2,1}\right)\right. \\
\quad-\left(c_{11} v_{1,1} \bar{v}_{1,1}+c_{22} v_{2,2} \bar{v}_{2,2}+c_{12}\left(v_{1,1} \bar{v}_{2,2}+\bar{v}_{1,1} v_{2,2}\right)+c_{55} v_{3,1} \bar{v}_{3,1}\right. \\
\left.\quad+c_{44} v_{3,2} \bar{v}_{3,2}\right)+i \omega M_{\alpha, \beta}\left(T \bar{v}_{\alpha, \beta}-\bar{T} v_{\alpha, \beta}\right) \\
\left.\quad+\frac{1}{\theta_{0}}\left(K_{33} T_{, 3} \bar{T}_{, 3}-K_{\alpha \beta} T_{, \alpha} \bar{T}_{, \beta}\right)+\omega^{2}\left(\rho v_{s} \bar{v}_{s}+\frac{c}{\theta_{0}} T \bar{T}\right)\right] d a
\end{gathered}
$$

In the limit case when $\beta \rightarrow 0$, we have

$$
\lim _{\beta \rightarrow 0}\left(1+\frac{\beta}{\alpha} x_{3}\right)^{-\frac{1}{\beta}}=e^{-\frac{x_{3}}{\alpha}}
$$

and the estimates (94), (97) and (98) are of exponential type, that is like those established in the low frequency range.

From Theorem 3, we observe that the coefficient of $x_{3}$ in the definition (75) of $I\left(x_{3}\right)$, that is the term $\mathscr{F}$, is independent of $x_{3}$ and hence its value can be estimated on a particular cross section. Therefore, for harmonic in time vibrations making $\mathscr{F}=0$ the analysis of the above section allows us to take $\beta=0$ and the decay and grow estimates expressed in (94), (97) and (98) are of exponential type. Concluding, there can be a set of harmonic vibrations for which we can predict an exponential decay or growth of the amplitudes. However, such class cannot be explicitly described by means of the given data. 
On the other hand, it is not immediately obvious how the amplitude $I(0)$ appearing in the decay estimates (97) and (98), depends upon data to the problem, and the absence of an explicit expression diminishes the practical applicability of the decay and growth estimates. However, such bounds can be obtained by means of the procedures used by Quintanilla and Straughan [15].

We have to emphasize that our results in this paper are related to recent results established by Chiriţă et al. [24] and Galeş and Chiriţă [25] where the exponential decay estimates for the amplitude of the steady-state vibrations are obtained without any restriction upon the frequency of vibrations.

Since the present results are established under strong ellipticity assumptions for the thermoelastic coefficients they are valuable for a large class of materials including those with negative Poisson's ratio (see, for example, Lakes [26]).

\section{REFERENCES}

1. D. S. Chandrasekharaiah, Hyperbolic Thermoelasticity: A Review of Recent Literature, Appl. Mech. Rev., vol. 51, pp. 705-729, 1998.

2. R. B. Hetnarski and J. Ignaczak, Generalized Thermoelasticity, J. Thermal Stresses, vol. 22, pp. 451-470, 1999.

3. R. B. Hetnarski and J. Ignaczak, Nonclassical Dynamical Thermoelasticity, Int. J. Solids Struct., vol. 37, pp. 215-224, 2000.

4. A. E. Green and P. M. Naghdi, Thermoelasticity Without Energy Dissipation, J. Elasticity, vol. 31, pp. 189-208, 1993.

5. A. E. Green and P. M. Naghdi, On Thermodynamics and the Nature of the Second Law, Proc. Roy. Soc. London A, vol. 357, pp. 253-270, 1977.

6. A. E. Green and P. M. Naghdi, A Unified Procedure for Contruction of Theories of Deformable Media I. Classical Continuum Physics II. Generalized Continua, III. Mixtures of Interacting Continua, Proc. Royal Society London A, vol. 448, pp. 335-356, pp. 357-377, pp. 379-388, 1995.

7. L. Nappa, Spatial Decay Estimates for the Evolution Equations of Linear Thermoelasticity Without Energy Dissipation, Journal of Thermal Stresses, vol. 21, pp. 581-592, 1998.

8. R. Quintanilla, On the Spatial Behavior in Thermoelasticity Without Energy Dissipation, Journal of Thermal Stresses, vol. 22, pp. 213-224, 1999.

9. D. S. Chandrasekharaiah, A Note on the Uniqueness of Solution in the Linear Theory of Thermoelasticity Without Energy Dissipation, J. Elasticity, vol. 43, pp. 279-283, 1996.

10. D. Iesan, On the Theory of Thermoelasticity Without Energy Dissipation, J. Thermal Stresses, vol. 21, pp. 295-307, 1998.

11. R. Quintanilla, On Existence in Thermoelasticity Without Energy Dissipation, J. Thermal Stresses, vol. 25, pp. 195-202, 2002.

12. R. Quintanilla, Damping of End Effects in a Thermoelastic Theory, Appl. Math. Letters, vol. 14, pp. 137-141, 2001.

13. R. Quintanilla, Convergence and Structural Stability in Thermoelasticity, Appl. Math. Computation, vol. 135, pp. 287-300, 2003.

14. R. Quintanilla and B. Straughan, Growth and Uniqueness in Thermoelasticity, Proc. Royal Society London A, vol. 456, pp. 1419-1429, 2000.

15. R. Quintanilla and B. Straughan, Energy Bounds for some Non-Standard Problems in Thermoelasticity, Proc. Royal Society London A., vol. 461, pp. 1147-1162, 2005. 
16. S. Chiriţă and M. Ciarletta, Spatial Evolution of Harmonic Vibrations in Linear Elasticity, Journal of Mechanics of Materials and Structures, vol. 3, no. 9, pp. 1675-1693, 2008.

17. S. Chiriţă, M. Ciarletta, and B. Straughan, Structural Stability in Porous Elasticity, Proc. R. Soc. Lond. A, vol. 462, pp. 2593-2605, 2006.

18. S. Chiriţă and M. Ciarletta, On the Structural Stability of Thermoelastic Model of Porous Media, Math. Methods Appl. Sci., vol. 31, pp. 19-34, 2008.

19. S. Chiriţă, Spatial Decay Estimates for Solutions Describing Harmonic Vibrations in a Thermoelastic Cylinder, J. Thermal Stresses, vol. 18, pp. 421-436, 1995.

20. J. N. Flavin and R. J. Knops, Some Spatial Decay Estimates in Continuum Dynamics, J. Elasticity, vol. 17, pp. 249-264, 1987.

21. J. N. Flavin, R. J. Knops, and L. E. Payne, Energy Bounds in Dynamical Problems for a Semi-infinite Elastic Beam, in G. Eason and R. W. Ogden (eds.), Elasticity: Mathematical Methods and Applications, pp. 101-112, Ellis-Horwood (Wiley), Chichester, 1990.

22. S. Chiriţă and M. Ciarletta, On the Spatial Behavior in Thermoelasticity Without Energy Dissipation, Journal of Thermal Stresses, vol. 32, pp. 1198-1214, 2009.

23. S. Chiriţă, A. Danescu, and M. Ciarletta, On the Strong Ellipticity of the Anisotropic Linearly Elastic Materials, J. Elasticity, vol. 87, pp. 1-27, 2007.

24. S. Chiriţă, C. Galeş, and I. D. Ghiba, On Spatial Behavior of the Harmonic Vibrations in Kelvin-Voigt Materials, J. Elasticity, vol. 93, pp. 81-92, 2008.

25. C. Galeş and S. Chiriţă, On Spatial Behavior in Linear Viscoelasticity, Quarterly of Applied Mathematics, 67, 2009, online published at http://www.ams.org/distribution/ qam/0000-000-00/S0033-569X-09-01149-0/.

26. R. S. Lakes, Foam Structures with a Negative Poisson's Ratio, Science, vol. 235, pp. 1038-1040, 1987. 